\title{
A PROSPECTIVE STUDY ON DRUG UTILIZATION PATTERN OF ANTIDEPRESSANTS IN NON-PSYCHIATRIC DEPARTMENTS IN A TERTIARY CARE TEACHING HOSPITAL
}

\author{
ANCY SEBASTIAN, ANEESHA PK, JOSHWA AREECKAL, SONIYA DAVIS*
}

Doctor of Pharmacy, St. James College of Pharmaceutical Sciences, Chalakudy, Kerala, India. Email: soniyadavis169@gmail.com

Received: 29 September 2018, Revised and Accepted: 29 September 2018

ABSTRACT

Objective: The aim of this study is to evaluate the pattern of prescription and utilization of antidepressants (ADs) for various non-psychiatric indications in department of general medicine, cardiology, surgery, gynecology, and orthopaedic of a tertiary care teaching hospital.

Methods: A prospective cross-sectional unicentric drug utilization study of inpatients was carried out in a 450 bedded tertiary care hospital. The study included 200 subjects admitted for both psychiatric and non-psychiatric illness. Pediatric patients and those with a length of stay <48 h were excluded from the study.

Results: Prescriptions of 200 patients were studied in which $55 \%$ were males and $45 \%$ were females. The most widely prescribed drug is alprazolam comprising $50 \%$ of the total drug consumption and is followed by clonazepam (19.5\%), amitriptyline (13.5\%), diazepam (9.5\%), escitalopram $(3.5 \%)$, lorazepam (3\%), and zolpidem $(0.5 \%)$. About $36 \%$ of the drugs were prescribed in the general medicine department, and $27.5 \%$ of the prescriptions were from the cardiology department. Prescriptions from surgery, orthopedics, and gynecology were $16 \%, 11.5 \%$, and $9 \%$ of the total, respectively.

Conclusion: ADs were found to be potentially useful for non-psychiatric indications such as peripheral and diabetic neuropathy, gastroenterological conditions, and urological conditions. No benefit was observed in insomnia and musculoskeletal conditions except for fibromyalgia. ADs were also found to improve the treatment outcome and quality of life of patients having comorbid psychiatric conditions being treated for non-psychiatric disorders.

Keywords: Antidepressants, Depression, Amitriptyline, Alprazolam, Tricyclic antidepressants, Selective serotonin reuptake inhibitors.

(c) 2019 The Authors. Published by Innovare Academic Sciences Pvt Ltd. This is an open access article under the CC BY license (http://creativecommons. org/licenses/by/4. 0/) DOI: http://dx.doi.org/10.22159/ajpcr.2019.v12i3.30004

\section{INTRODUCTION}

Antidepressants (ADs) are the widely prescribed medication among the general population. Prevalence of their 1 year consumption ranges from $6 \%$ to $10 \%$ [1]. ADs are mainly used for the management of major depressive episodes and anxiety [2]. Between 1980 and 2008, their utilization has risen by sevenfold in France, from €84 million to $€ 525$ million per year [3]. Selective serotonin reuptake inhibitors (SSRIs) and selective norepinephrine reuptake inhibitors (SNRIs) topped the chart of drug sales worldwide. ADs are the third most consumed medication in the United States of America [4]. Alarming surge in the utilization of these agents is a source of concern for economists although the exact reason behind this trend remains unknown $[5,6]$. Polypharmacy in geriatrics also pose a major concern, and some individual-based studies point out that amitriptyline and alprazolam are widely used as sedatives in the elderly despite of their strong anti-cholinergic action [7]. According to various studies, there are two probable explanations; the first is over-prescription for psychiatric disorders. Evidence suggests that ADs are not capable of producing dramatic changes in moderate-tosevere episodes; however, they are life changers in mild depression $[8,9]$. Sometimes they are stopped too early or given too long. The second reason is the prescription of them for "non-psychiatric conditions." Evaluating their potential merits are demanding, as they are extensively used for numerous non-psychiatric illness including gastroenterological and neurological an urological diseases.

Emerging evidence substantiates that ADs are more often than not prescribed for health disorders outside the field of psychiatry [10]. This rate, according to various observational studies, ranges from $25 \%$ to $60 \%$. Research has pointed out that, apart from non-psychiatric conditions, these drugs are also given for a number of off-label uses. Mostly, personal feelings of health practitioners outweigh ADs prescription rather than relevant scientific evidence.

The aim of this study is to review evidence from various guidelines of AD prescription for non-psychiatric conditions in a tertiary care setting. The rationale behind this study is to make the health-care team aware about the unanticipated consequences from the overuse of $\mathrm{AD}$ class of medications and monitor their use in the hospital settings.

\section{METHODS}

This was an observational, cross-sectional study conducted in a tertiary care teaching hospital, India. The study was carried out for a period of 6-month after approval of the Institutional Ethics Committee of Hospital (IHEC, SJH). The protocol was approved by the committee with the approval number SJPCEC/P25/PP/2014/033 and the hospital approval number SJCP/DIR/A.19/2015-2016. A total of 200 patients were enrolled in this research.

\section{Inclusion criteria}

The following criteria were included in the study:

- All inpatients in cardiology, general medicine, orthopedics, surgery, gynecology departments during the prescribed study period.

- All inpatients managed with AD drugs

- Age: 25-75 years

- Gender: Both females and males.

\section{Exclusion criteria}

The following criteria were excluded from the study:

- Pediatric patients

- Patients with a length of stay $>48 \mathrm{~h}$. 
Data of patients meeting the inclusion criteria were promptly recorded in the predesigned data pro forma by attending the daily ward rounds, reviewing patient medications, collecting relevant details from patients and carrying out the AD utilization review. Prescriptions obtained were evaluated for demographic data using the WHO core drug prescription indicators.

\section{RESULTS}

A prospective observational study was carried out for a period of 6 months in a 450 bedded tertiary care hospital to evaluate the drug utilization pattern of ADs in non-psychiatric departments. A total of 200 patients from five wards were enrolled in the study based on inclusion criteria. The results obtained were as follows;

Patients of age group 25-75 years were selected, and the majority belongs to the group of $65-75$ years $(33 \%)$ of which $55 \%$ were males and $45 \%$ were females.

Table 1 presents that, among various ADs prescribed, alprazolam pecked the list (50.5\%), followed by clonazepam (19.5\%), amitriptyline (13.5\%), diazepam (9.5\%), escitalopram (3.5\%), and lorazepam (3\%), and the least prescribed drug during the study period was zolpidem $(0.5 \%)$

Table 2 shows that out of all cases $(n=200)$ AD use was the maximum in the department of general medicine (27.5\%), and the least was in the gynecology department (9\%)

In the cardiology department, the most prescribed drug was alprazolam 52\% ( $\mathrm{n}=29)$ and the least was escitalopram 3.6\% $(\mathrm{n}=20)$ while in orthopedics amitriptyline $56.5 \%(n=13)$ was the topper and clonazepam was given the least $8.6 \%(n=2)$. Alprazolam was the most utilized ADs in the department of general medicine comprising $45 \%$ of prescription $(\mathrm{n}=32)$. Diazepam and zolpidem shared the lowest position with $1.3 \%$ each $(\mathrm{n}=1)$. In the surgery ward, $90.6 \%$ of total prescription was for alprazolam (n-29) and the amitriptyline was the least prescribed one. Diazepam was the only $(n=18) A D$ used in the obstetrics and the gynecology department.

Fig. 1 shows the percentage distribution of antidepressants for the management of psychiatric illness in various departments. Out of samples $(n=23)$, about $4 \%(n=8)$ of drug usage in cardiac patients were for the management of underlying psychiatric conditions. Similar prescribing pattern was found in the general medicine ward $5 \%(n=10)$ and orthopedic ward $2.5 \%(n=5)$.

Fig. 2 shows that about 177 cases for non-psychiatric illness were studied ( $\mathrm{n}=177$ ), $20 \%$ of antidepressant were used for treating primary

Table 1: Distribution of ADs in prescriptions

\begin{tabular}{ll}
\hline Medication & Frequency (\%) \\
\hline Alprazolam & $101(50.5)$ \\
Clonazepam & $39(19.5)$ \\
Amitriptyline & $27(13.5)$ \\
Lorazepam & $27(3)$ \\
Escitalopram & $7(3.5)$ \\
Zolpidem & $1(0.5)$ \\
Diazepam & $19(9.5)$ \\
\hline
\end{tabular}

ADs: Antidepressants

Table 2: Distribution based on department

\begin{tabular}{ll}
\hline Department & Frequency (\%) \\
\hline Cardiology & $55(27.5)$ \\
General medicine & $72(36)$ \\
Orthopedics & $23(11.5)$ \\
Surgery & $32(16)$ \\
Gynecology & $18(9)$ \\
\hline
\end{tabular}

insomnia $(n=40)$, followed by pre-operative and post-operative pain management with $13.5 \%(n=27)$ and $13 \%(n=26)$, respectively, followed by diabetic and peripheral neuropathy $12.5 \%(\mathrm{n}=25)$, inflammatory bowel syndrome $5 \%$, overactive bladder $5 \%$, urinary incontinence $4 \%$, and panhypopituitarism $3 \%$.

Depression is one of the most prevalent risk factors for the development and progression of coronary artery disease, cardiovascular disease, diabetes mellitus, etc. Apart from its indicated use, ADs may also be given to improve the treatment outcome and patients quality of life. Distribution of AD based on a comorbid psychiatric condition in nonpsychiatric departments was analyzed.

\section{DISCUSSION}

Drug utilization studies (DUS) are a tool for assessing the prescribing, dispensing, and distribution of drugs. DUS is mainly intended to facilitate rational use of medicines [11].

ADs are drugs implied in the treatment of depression and to prevent its reoccurrence. Apart from their primary indications, they are widely being used for various non-psychiatric conditions. This study was designed to evaluate the need of ADs medication for these indications with evidence for its benefit in every category. In this study, a total of 200 prescriptions were analyzed, of which, 109 (55\%) were males and $91(45 \%)$ were females which are in concordance with the study conducted by Kumar et al. [12]

Amitriptyline is the only AD used in prophylaxis of vascular headaches $(n=5)$. Increased use of amitriptyline may be because the current recommendations consider it as level IA evidence drug, with a

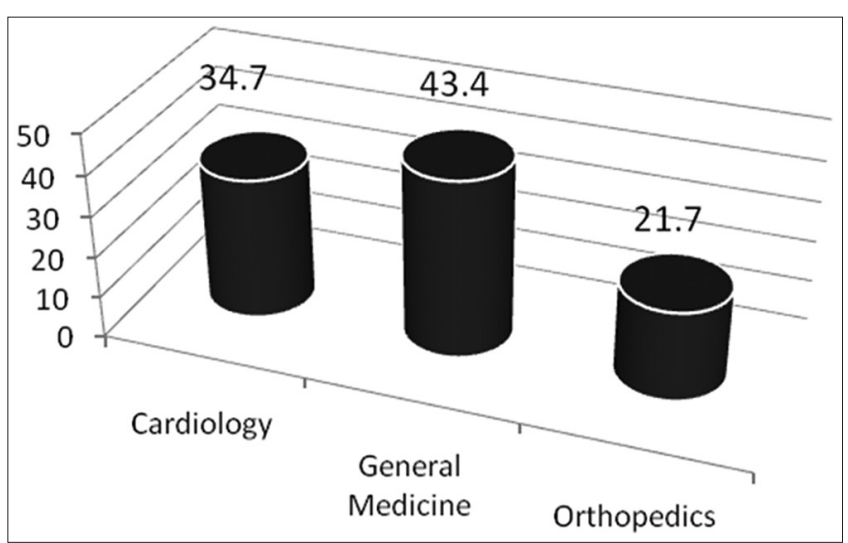

Fig. 1: Percentage distribution based on psychiatric indication

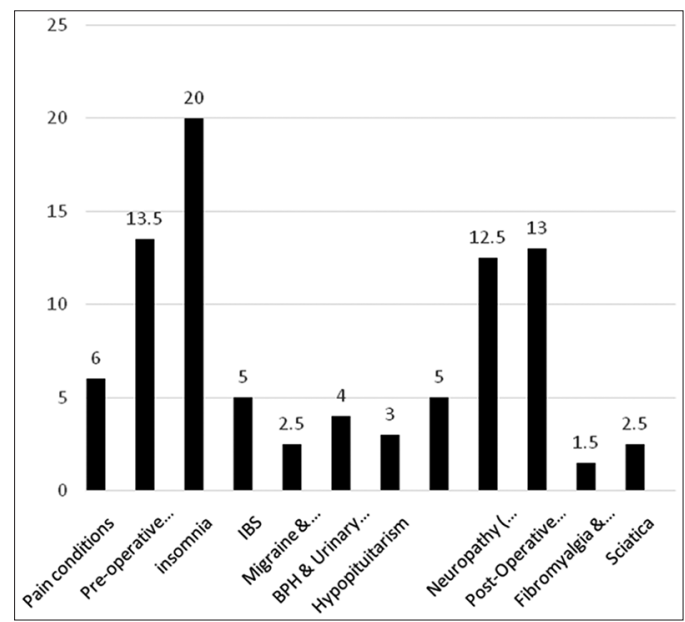

Fig. 2: Percentage distribution based on non-psychiatric indication 
therapeutic dose ranging from 30 to $150 \mathrm{mg} /$ day. ADs were also beneficial in gastroenterological conditions like IBS. Tricyclic ADs (TCA) could be used as a second line agent and SSRI as third-line agent. It aids to control the symptoms of IBS such as burping and persistent abdominal pain. We have found that low dose amitriptyline was rarely used in the treatment of GERD as that demonstrated by Morgan et al. that the doses for the pain-relieving and neuromodulatory effects of the drug were below the minimum therapeutic dose range [13] As the disease increase in severity, higher doses may be needed to modify the deteriorating gastrointestinal and bowel functions.

ADs were used for fibromyalgia in $1.5 \%$ of cases in our study as substantiated by Hauser et al. in the study "treatment of fibromyalgia syndrome with $\mathrm{AD}$ " a meta-analysis in which $\mathrm{AD}$ medications can help with the remission of pain, lethargy, insomnia, and improvement of health-related quality of life in patients with fibromyalgia [14].

ADs should be used as first-line agent for peripheral neuropathy and diabetic neuropathy which results in the frequently disabling pain. ADs are highly beneficial for neuropathic pain due to its analgesic actions. A prescription pattern study by Jena et al. found out that TCA and SNRI need to be given for neuropathic pain instead of adding nonsteroidal anti-inflammatory drugs and opioids [15]. TCAs are most effective and are considered to as first-line agents while SNRIs are second-line agents for the treatment of neuropathic pain. We have found 37 cases of patients being treated with ADs in various departments for pain in which $12.5 \%$ is for peripheral and diabetic neuropathy and $6 \%$ for other pain conditions wherein TCA were the most deployed drug class. A study by pinky Dharmshaktu et al. reveals that TCA was the gold standard drug for the control of persistent neuropathic pain [16].

In orthopedics patients, alprazolam is highly potent in decreasing the post-operative analgesic use, and it also improves knee functions by reducing pain threshold particularly in patients undergoing TKR and improves quality of life for those with concurrent depression. We havefound $21.7 \%$ cases with concomitant mental status alterations in this department which is in contrast to the $56 \%$ of cases in a study by Yilmaz et al. [17]. The difference may be attributed to a probably higher level of patient mental status assessment done in the latter in comparison to a tertiary care hospital with limited prospects.

ADs, especially SSRI contribute to hyponatremia. One such case of a patient diagnosed with sodium depletion with relevant laboratory evidence and given the drug escitalopram was found during the research which can be substantiated by the observations of Grover et al., revealing a higher incidence of SSRI associated electrolyte abnormality drawing a conclusion that TCA can be a more safer choice [18].

\section{CONCLUSION}

By analyzing the data collected from the study, two patterns of prescribing anti-depressants were found in non-psychiatric wards; prescribing for treating non-psychiatric conditions such as neuropathy, IBS, and urological conditions and prescribing to treat comorbid psychiatric illness which may pose a risk for the non-psychiatric condition for which the patient is being treated.

ADs were found to be highly beneficial in the treatment of pain and neuropathic conditions. Unnecessarily prescribing and consuming of ADs may lead to addiction. The certain patient tends to take the drug even after the recommended course got over. ADs are drugs which have to be prescribed if and only if, it is indicated and necessary. Evidence against prescribing them was also found. The overall incongruity of the gathered data impeded the critical evaluation of the evidence. It is also crucial to spotlight the difference between appraising the scientifically acquainted effects of an $\mathrm{AD}$ for a specific condition and their potential advantages at the right dose and right time for a specific patient despite a lower level of proof.

\section{AUTHORS' CONTRIBUTIONS}

Ancy Sebastian: Prepared a framework for the manuscript. Aneesha PK: Conceptualized the article, developed the protocol for the manuscript. Joshwa Areeckal: Reviewed and edited the manuscript. Soniya Davis: Performed the literature search, drafted the manuscript

\section{CONFLICTS OF INTEREST}

Authors declared that they have no conflicts of interest.

\section{REFERENCES}

1. Gasquet I, Nègre-Pagès L, Fourrier A, Nachbaur G, El-Hasnaoui A, Kovess $\mathrm{V}$, et al. Psychotropic drug use and mental psychiatric disorders in France; Results of the general population ESEMeD/MHEDEA 2000 epidemiological study. Encephale 2005;31:195-206.

2. Olfson M, Marcus SC. National patterns in antidepressant medication treatment. Arch Gen Psychiatry 2009;66:848-56.

3. Ventes de Médicaments aux Officineset aux Hôpitaux en France: Chiffresclés 2010. [Sales of Medicines to Pharmacies and Hospitals in France: Key Figures 2010]. Rapport D'Expertise.

4. Alonso J, Angermeyer MC, Bernert S, Bruffaerts R, Brugha TS, Bryson $\mathrm{H}$, et al. Psychotropic drug utilization in Europe: Results from the European study of the epidemiology of mental disorders (ESEMeD) project. Acta Psychiatr Scand Suppl 2004;109:55-64

5. Grolleau A, Cougnard A, Bégaud B, Verdoux H. Psychotropic drug use and correspondence with psychiatric diagnoses in the mental health in the general population survey. Encephale 2008;34:352-9.

6. Piek E, van der Meer K, Hoogendijk WJ, Penninx BW, Nolen WA. Most antidepressant use in primary care is justified; Results of the Netherlands study of depression and anxiety. PLoS One 2011;6:e14784.

7. Gopinath S, Rajalingam B, Sriram S, Vijayakumar S. An individual based study of the geriatric population: A polypharmacy. Int J Pharm Pharm Sci 2013;3:63-6.

8. Arroll B, Elley CR, Fishman T, Goodyear-Smith FA, Kenealy T, Blashki $G$, et al. Antidepressants versus placebo for depression in primary care. Cochrane Database Syst Rev 2009;3:CD007954.

9. Stewart JA, Deliyannides DA, Hellerstein DJ, McGrath PJ, Stewart JW Can people with nonsevere major depression benefit from antidepressant medication? J Clin Psychiatry 2012;73:518-25.

10. Mojtabai R, Olfson M. Proportion of antidepressants prescribed without a psychiatric diagnosis is growing. Health Aff (Millwood) 2011;30:1434-42.

11. Viswanathan N, Gandhi IS, Shashindran CH, Adithan C. Drug utilisation study of antimicrobial agents. Indian J Med Res 1981;74:772-8.

12. Mercier A, Auger-Aubin I, Lebeau JP, Schuers M, Boulet P, Hermil JL, et al. Evidence of prescription of antidepressants for non-psychiatric conditions in primary care: An analysis of guidelines and systematic reviews. BMC Fam Pract 2013;14:55.

13. Morgan V, Pickens D, Gautam S, Kessler R, Mertz H. Amitriptyline reduces rectal pain related activation of the anterior cingulate cortex in patients with irritable bowel syndrome. Gut 2005;54:601-7.

14. Häuser W, Bernardy K, Uçeyler N, Sommer C. Treatment of fibromyalgia syndrome with antidepressants: A meta-analysis. JAMA 2009;301:198-209.

15. Jena SS, Jena M, Patro N, Mishra S, Panda M, Dash M. Patterns of prescription and ADR monitoring of drugs in the management of neuropathic pain in a tertiary care teaching hospital. Int J Pharm Pharm Sci 2014;6:246-51.

16. Dharmshaktu P, Tayal V, Kalra BS. Efficacy of antidepressants as analgesics: A review. J Clin Pharmacol 2012;52:6-17.

17. Yılmaz B, Kömür B, Aktaş E, Sonnur Yılmaz F, Copuroğlu C, Özcan M, et al. Impact of alprazolam on comorbid pain and knee functions in total knee arthroplasty patients diagnosed with anxiety and depression. Open Orthop J 2015;9:530-5.

18. Grover S, Biswas P, Bhateja G, Kulhara P. Escitalopram-associated hyponatremia. Psychiatry Clin Neurosci 2007;61:132-3. 